\title{
Geriatric comanagement reduces perioperative complications and shortens duration of hospital stay after lumbar spine surgery: a prospective single-institution experience
}

\author{
Presented at the 2017 AANS/CNS Joint Section on Disorders of the Spine and Peripheral Nerves \\ Owoicho Adogwa, MD, MPH, ${ }^{1}$ Aladine A. Elsamadicy, BE, ${ }^{2}$ Victoria D. Vuong, MS, ${ }^{1}$ \\ Jessica Moreno, MS, BSN, RN, ${ }^{4}$ Joseph Cheng, MD, MS, ${ }^{3}$ Isaac O. Karikari, MD, ${ }^{2}$ and \\ Carlos A. Bagley, MD, MBA ${ }^{4}$
}

\begin{abstract}
1Department of Neurosurgery, Rush University Medical Center, Chicago, Illinois; ²Department of Neurosurgery, Duke University Medical Center, Durham, North Carolina; ${ }^{3}$ Department of Neurosurgery, Yale University, New Haven, Connecticut; and ${ }^{4}$ Department of Neurosurgery, University of Texas Southwestern, Dallas, Texas
\end{abstract}

OBJECTIVE Geriatric patients undergoing lumbar spine surgery have unique needs due to the physiological changes of aging. They are at risk for adverse outcomes such as delirium, infection, and iatrogenic complications, and these complications, in turn, contribute to the risk of functional decline, nursing home admission, and death. Whether preoperative and perioperative comanagement by a geriatrician reduces the incidence of in-hospital complications and length of inhospital stay after elective lumbar spine surgery remains unknown.

METHODS A unique model of comanagement for elderly patients undergoing lumbar fusion surgery was implemented at a major academic medical center. The Perioperative Optimization of Senior Health (POSH) program was launched with the aim of improving outcomes in elderly patients (> 65 years old) undergoing complex lumbar spine surgery. In this model, a geriatrician evaluates elderly patients preoperatively, in addition to performing routine preoperative anesthesia surgical screening, and comanages them daily throughout the course of their hospital stay to manage medical comorbid conditions and coordinate multidisciplinary rehabilitation along with the neurosurgical team. The first 100 cases were retrospectively reviewed after initiation of the POSH protocol and compared with the immediately preceding 25 cases to assess the incidence of perioperative complications and clinical outcomes.

RESULTS One hundred twenty-five patients undergoing lumbar decompression and fusion were enrolled in this pilot program. Baseline characteristics were similar between both cohorts. The mean length of in-hospital stay was $30 \%$ shorter in the POSH cohort (6.13 vs 8.72 days; $p=0.06$ ). The mean duration of time between surgery and patient mobilization was significantly shorter in the POSH cohort compared with the non-POSH cohort (1.57 days vs 2.77 days; $p=$ $0.02)$, and the number of steps ambulated on day of discharge was 2-fold higher in the POSH cohort ( $p=0.04)$. Compared with the non-POSH cohort, the majority of patients in the POSH cohort were discharged to home (24\% vs $54 \%$; $p=0.01)$.

CONCLUSIONS Geriatric comanagement reduces the incidence of postoperative complications, shortens the duration of in-hospital stay, and contributes to improved perioperative functional status in elderly patients undergoing elective spinal surgery for the correction of adult degenerative scoliosis.

https://thejns.org/doi/abs/10.3171/2017.5.SPINE17199

KEY WORDS comanaged care; spine surgery; complications; length of stay; perioperative care; geriatric; lumbar

ABBREVIATIONS AFib = atrial fibrillation; $\mathrm{BMI}=$ body mass index $\mathrm{CAD}=$ coronary artery disease $\mathrm{CHF}=$ congestive heart failure; $\mathrm{CVD}=$ cardiovascular disease; $\mathrm{DM}=$ diabetes mellitus; HTN = hypertension; IQR = interquartile range; LOS = length of stay; MI = myocardial infarction; POSH = Perioperative Optimization of Senior Health; SNF $=$ skilled nursing facility.

SUBMITTED February 12, 2017. ACCEPTED May 3, 2017.

INCLUDE WHEN CITING Published online September 29, 2017; DOI: 10.3171/2017.5.SPINE17199. 
$\mathrm{D}$ EGENERATIVE disorders of the lumbar spine are a major cause of low-back pain and morbidity in the rapidly growing geriatric population in the United States. ${ }^{13,18,20,22,23}$ Symptomatic low-back and leg pain can lead to loss of function or the inability to perform basic activities of daily living. Surgical treatment for low-back pain historically has been pursued with limited success in elderly patients; however, the need and demand for surgical treatment in this population have been increasing to reduce pain and disability and to increase quality of life. ${ }^{3,7,20}$

Geriatric patients undergoing complex spine surgery have unique needs that must be considered. Due to the physiological changes of aging and associated medical comorbidities, elderly patients have a higher risk of perioperative complications and adverse events including delirium, infection, and other iatrogenic complications. These issues ultimately can contribute to functional decline, nursing home admissions, and death. Geriatricians, who are skilled in caring for the clinical and social complexities of the elderly population, may be able to identify and reduce these risks perioperatively. Accordingly, comanagement by both neurosurgeons and geriatricians could offer a way to optimize medical management and improve outcomes for geriatric patients undergoing spinal surgery. However, whether neurosurgical-geriatric comanagement empirically improves the patient's perioperative experience or reduces complications is unknown.

The aim of this study was to determine if neurosurgical-geriatric comanagement significantly improves postoperative care and decreases perioperative complications, in-hospital length of stay (LOS), and 30-day readmission rates.

\section{Methods \\ Patient Selection}

This was a retrospective study of 125 elderly patients undergoing elective spinal surgery at a major academic medical center following implementation of the Perioperative Optimization of Senior Health (POSH) program. The records of the first 100 cases following POSH implementation and the records of the immediately preceding 25 cases were reviewed.

We included patients aged 65 years and older with the following characteristics: 1) had back pain and/or radiculopathy; 2) radiographic evidence of thoracolumbar deformity; 3) prior failed nonsurgical treatment; and 4) underwent multilevel lumbar decompression and fusion. Patients were excluded if they had a severe coexisting pathology that could confound the assessment of operative outcome or were nonambulatory at baseline.

Demographic variables that were evaluated included patient age, sex, and body mass index (BMI). Comorbidities included hypertension (HTN), diabetes mellitus (DM), cardiovascular disease (CVD), coronary artery disease (CAD), congestive heart failure (CHF), atrial fibrillation (AFib), and prior myocardial infarction (MI). Smoking status was also determined.

\section{Geriatric Comanagement Model}

A unique model of shared care for elderly patients un- dergoing lumbar fusion surgery was implemented at a major academic medical center. The POSH program was launched with the aim of improving outcomes in elderly patients ( $>65$ years old) undergoing complex spine surgery procedures. In addition to routine preoperative anesthesia surgical screening, this model utilizes a geriatrician to evaluate patients preoperatively and postoperatively. In addition, the geriatrician comanages the patients throughout the course of their hospital stay to manage medical comorbidities and coordinate multidisciplinary rehabilitation with the neurosurgical team.

\section{Postoperative Complications}

We assessed the postoperative complications of each patient included in the study. Surgical complications were defined as complications that were the direct result of surgery. These complications included hardware failure requiring a revision procedure, surgical site infections, and new-onset sensory/motor deficits. Nonsurgical complications were defined as complications resulting from medical conditions not directly related to surgery. These complications included deep vein thrombosis, pulmonary embolism, MI, chest pain, fever, and other medical complications.

\section{0-Day Hospital Readmission Rate}

We reviewed the hospital charts of each readmission to determine the cause of readmission. Unplanned readmissions were defined as either surgical or nonsurgical complications. Planned readmissions were defined as either a staged or rescheduled procedure. For a staged procedure, the patient was discharged with the expectation that he or she would be readmitted for the subsequent stage of the procedure. A rescheduled procedure occurred when the patient was admitted on the day of surgery but the procedure was cancelled prior to surgery, the patient was discharged, and the procedure was rescheduled to occur within a month. Planned readmissions were not included in the final data analysis and were not used to calculate surgical readmission rates. Only unplanned hospital readmissions were included in the final analysis.

\section{Postoperative Functional Status}

Variables included to assess postoperative functional status included the duration to first ambulation, the distance ambulated on the first day of ambulation, and the distance ambulated on the day of discharge. The in-hospital LOS and discharge disposition (home vs skilled nursing facility $[\mathrm{SNF}]$ vs acute rehabilitation facility) were also assessed.

\section{Statistical Analysis}

Parametric data were expressed as the mean \pm SD and compared using the Student t-test. Nonparametric data were expressed as the median (interquartile range [IQR]) and compared using the Mann-Whitney U-test. Nominal data were compared using the chi-square test. All tests were 2-sided and statistically significant if the $p$ value was less than 0.05. We used SAS 9.3 (SAS Institute, Inc.) for all data preparation and analysis. 
TABLE 1. Baseline characteristics of elderly patients undergoing lumbar fusion surgery

\begin{tabular}{lccc}
\hline Characteristic & POSH & Non-POSH & p Value \\
\hline No. of patients & 100 & 25 & \\
\hline Age, yrs & $73.60 \pm 6.03$ & $73.04 \pm 4.88$ & 0.62 \\
\hline BMl, kg/m & $28.89 \pm 6.24$ & $27.15 \pm 5.37$ & 0.17 \\
\hline Male & 41.00 & 36.00 & 0.65 \\
\hline Smoker & 3.00 & 16.00 & 0.10 \\
\hline CHF & 4.00 & 12.00 & 0.25 \\
\hline CAD & 21.00 & 28.00 & 0.49 \\
\hline CVD & 7.00 & 8.00 & 0.87 \\
\hline MI & 9.00 & 12.00 & 0.69 \\
\hline HTN & 62.00 & 64.00 & 0.85 \\
\hline AFib & 2.00 & 8.00 & 0.30 \\
\hline DM & 23.00 & 8.00 & 0.03 \\
\hline
\end{tabular}

Values are shown as the percentage or mean \pm SD unless stated otherwise.

\section{Results}

One hundred twenty-five adult patients ( 25 patients in the non-POSH cohort and 100 patients in the POSH cohort) were included in this study. There was no significant difference in the mean age between groups $(73.04 \pm 4.88$ years in the non-POSH cohort vs $73.60 \pm 6.03$ years in the POSH cohort; $\mathrm{p}=0.62$ ). An approximately equal percentage of men were included in both groups $(36.00 \%$ of the non-POSH cohort vs $41.00 \%$ of the POSH cohort; $\mathrm{p}=$ $0.65)$. There was no significant difference in BMI (27.15 \pm $5.37 \mathrm{~kg} / \mathrm{m}^{2}$ in the non-POSH cohort vs $28.89 \pm 6.24 \mathrm{~kg} / \mathrm{m}^{2}$ in the POSH cohort; $\mathrm{p}=0.17)$ or smoking status $(16.00 \%$ of the non-POSH cohort vs $3.00 \%$ of the POSH cohort were smokers; $p=0.10$ ) between cohorts. There were no significant differences in the prevalence of other comorbidities such as CHF, CAD, CVD, MI, HTN, AFib, or DM. A comparison of these baseline characteristics is shown in Table 1.

The median (IQR) number of fusion levels between groups was similar (6.5 [3-11] levels in the non-POSH cohort vs 6 [3-10] levels in the POSH cohort; $p=0.90$ ). The percentage of patients with intraoperative durotomy was similar between both groups $(12.00 \%$ of the non-POSH cohort vs $9.00 \%$ of the POSH cohort; $\mathrm{p}=0.68$ ). There were no incidences of nerve root injury or spinal cord injury in either group (Table 2).

\section{Postoperative Complications Profile}

The postoperative incidence of delirium was not significantly different between groups $(16.00 \%$ in the non-POSH cohort vs $18.00 \%$ in the POSH cohort; $\mathrm{p}=0.81$ ). The incidence of ileus was also similar between cohorts $(16.00 \%$ in the non-POSH cohort vs $10.00 \%$ in the POSH cohort; $\mathrm{p}$ $=0.46)$. The incidences of pneumonia $(4.00 \%$ in the nonPOSH cohort vs $5.00 \%$ in the POSH cohort; $\mathrm{p}=0.82$ ) and urinary tract infection $(4.00 \%$ in the non-POSH cohort vs $8.00 \%$ in the POSH cohort; $\mathrm{p}=0.41$ ) were not significantly different between groups. There were no incidences of postoperative deep vein thrombosis or pulmonary embo-
TABLE 2. Total and cohort-specific postoperative complication rates

\begin{tabular}{lccc}
\hline \multicolumn{1}{c}{ Variable } & POSH & Non-POSH & p Value \\
\hline Operative details/complications & & & \\
\hline No. of levels & $6(3-10)$ & $6.5(3-11)$ & 0.90 \\
\hline Intraop durotomy & 9.00 & 12.00 & 0.68 \\
\hline Nerve root injury & 0.00 & 0.00 & 1.00 \\
\hline Spinal cord injury & 0.00 & 0.00 & 1.00 \\
\hline Postoperative complications & & & \\
\hline Delirium & 18.00 & 16.00 & 0.81 \\
\hline Ileus & 10.00 & 16.00 & 0.46 \\
\hline Pneumonia & 5.00 & 4.00 & 0.82 \\
\hline Urinary tract infection & 8.00 & 4.00 & 0.41 \\
\hline Deep vein thrombosis & 1.00 & 0.00 & 0.31 \\
\hline Pulmonary embolism & 2.00 & 0.00 & 0.31 \\
\hline Ml & 1.00 & 1.00 & 0.47 \\
\hline
\end{tabular}

Values are presented as the percentage or median (IQR) unless stated otherwise.

lism in the non-POSH cohort, but 1 case of DVT and 2 cases of pulmonary embolism in the POSH cohort $(\mathrm{p}=0.31$ and $\mathrm{p}=0.31$, respectively). There was a similar incidence of MI in both groups $(1.00 \%$ in the non-POSH cohort vs $1.00 \%$ in the POSH cohort; $\mathrm{p}=0.47$ ). A comparison of these postoperative complications is shown in Table 2.

\section{Postoperative Functional Status}

Compared with the POSH cohort, the non-POSH cohort had a 2-fold increase in the number of days from surgery to ambulation $(2.77 \pm 2.28$ days in the non-POSH cohort vs $1.57 \pm 0.96$ days in the POSH cohort; $p=0.02$ ) (Fig. 1). The number of feet walked on ambulatory Day 1 was almost 2.5 times greater for the POSH group compared with the non-POSH group $(44.92 \pm 75.38$ feet in the non-POSH cohort vs $110.31 \pm 122.99$ feet in the POSH cohort; $p=0.01$ ) (Table 3). The number of feet walked on the day of discharge was also greater in the POSH group compared with the non-POSH group $(111.60 \pm 116.59$ feet in the non-POSH cohort vs $211.61 \pm 250.23$ feet in the POSH cohort; $p=0.04$ ) (Fig. 1).

The mean in-hospital LOS was approximately 2 days (30\%) shorter for the POSH group than the non-POSH group $(8.72 \pm 6.10$ days in the non-POSH cohort vs $6.13 \pm$ 5.73 days in the POSH cohort; $p=0.06$ ) (Fig. 1). The majority of patients in the POSH cohort were discharged directly home $(24.00 \%$ in the non-POSH cohort vs $54.00 \%$ in the POSH cohort; $\mathrm{p}=0.01$ ), with only a minority of patients requiring an additional stay at an SNF or acute rehabilitation facility $(20.00 \%$ in the non-POSH cohort vs $3.00 \%$ of the POSH cohort; $p=0.04$ ) (Fig. 1).

There was no significant difference in the 30-day readmission rate $(8.00 \%$ of the non-POSH cohort vs $10.00 \%$ of the POSH cohort; $p=0.77$ ) or 30 -day mortality rate $(0.00 \%$ of the non-POSH cohort vs $0.00 \%$ of the POSH cohort; $\mathrm{p}=0.99$ ) between groups. A comparison of these functional status outcomes is shown in Table 3. 

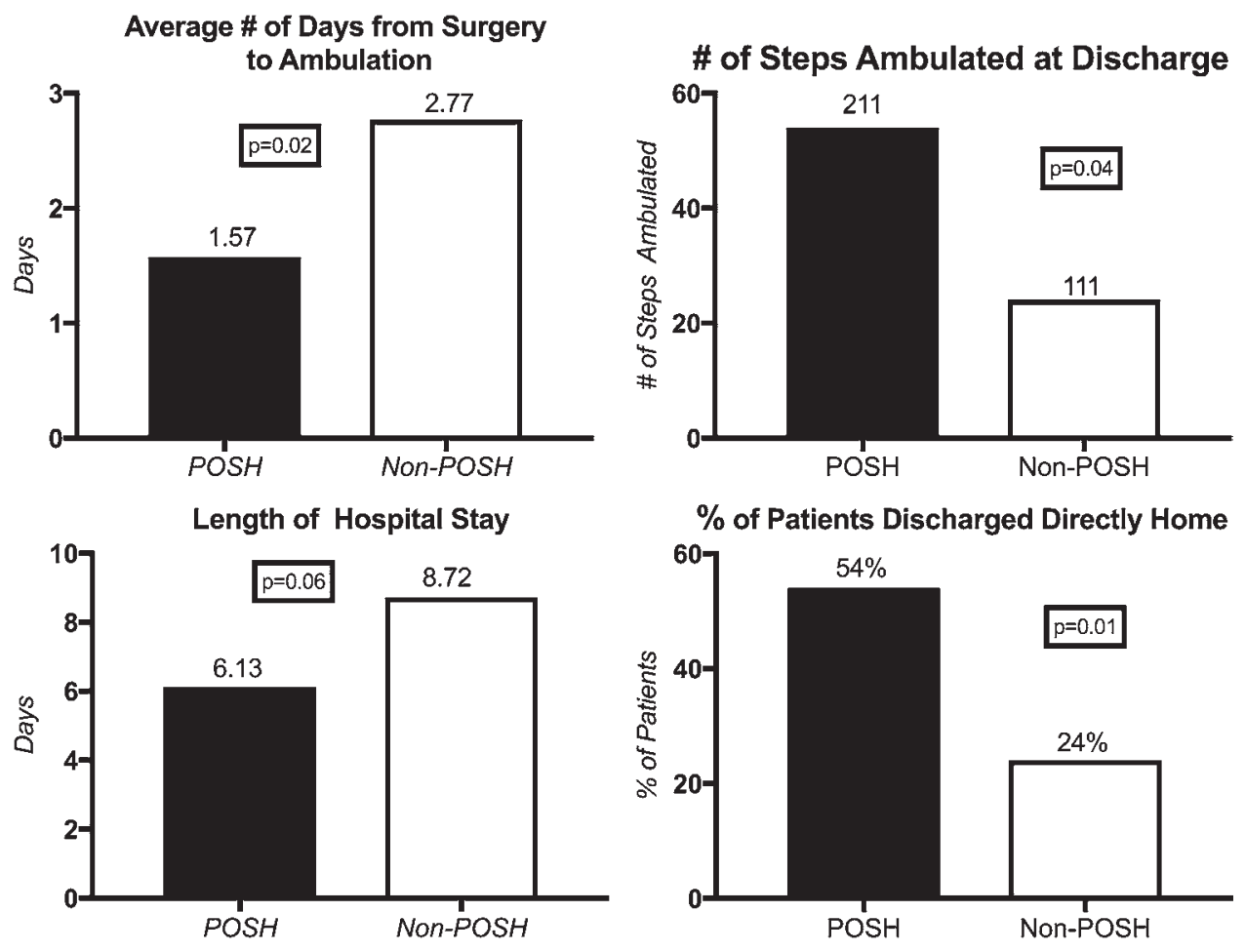

FIG. 1. Functional status of patients in the POSH cohort appears to be superior to that of non-POSH patients. The number of days from surgery to mobilization is significantly shorter. The majority of patients in the POSH cohort were discharged directly to home.

\section{Discussion}

In this retrospective cohort study of elderly patients $(>$ 65 years old) undergoing elective spinal surgery, we observed that patients who received neurosurgical-geriatric comanagement (POSH cohort) had a shorter in-hospital LOS and improved functional status at discharge. Patients participating in the POSH program were mobilized sooner and to a greater degree than non-POSH patients, and they were more likely to be discharged directly home.

Surgical management for the treatment of symptomatic low-back and leg pain is currently associated with more favorable outcomes than nonsurgical management. Recent studies have shown that surgical management of degenerative lumbar disorders can lead to improved quality of life in the geriatric population. In a retrospective study of 4370 patients undergoing lumbar spine surgery, McGirt et al. found that elderly patients experienced a similar improvement in pain, disability, and quality of life without an increase in complications or 90-day hospital readmission when compared with younger patients. ${ }^{17}$ Similarly, Devin et al. also found an equivalent improvement in pain, disability, and quality of life among elderly patients undergoing elective lumbar spinal surgery for degenerative disease. Furthermore, Devin et al. observed that the costeffectiveness, as measured by cost per quality-adjusted life year gained, of lumbar decompression with and without fusion was similar between 2 age groups. ${ }^{9}$ In another retrospective study of 69 elderly patients undergoing revision lumbar decompression and instrumented fusion, Adogwa et al. determined that revision lumbar surgery also leads to improvement in low-back pain, disability, and quality of life, while demonstrating improved cost-effectiveness over other nonoperative treatment modalities. ${ }^{1,2}$

Spinal surgery in elderly patients has been associated with an unfavorable complications profile. In a retrospective study of 453 elderly patients ( $>65$ years old) undergoing surgery for adult degenerative scoliosis, Drazin et al. determined that older age was associated with a higher overall reoperation rate, higher readmission rates, and more complications during index hospitalization and within 30 days of discharge. ${ }^{11}$ In another retrospective study of elderly patients in the Nationwide Inpatient Sample database who underwent surgery for adult degenerative scoliosis, Worley et al. found that elderly patients had an increased risk of inpatient morbidity and mortality compared with patients between the ages of 25 and 64 years. ${ }^{24}$ Daubs et al. found that increasing age and age over 69 years predicted complications in patients undergoing surgery for adult spinal deformities. ${ }^{8}$ Deyo et al. found that major medical complications and 30-day mortality rates are independently associated with older age. Additionally, Deyo et al. found that the likelihood of 30-day rehospitalization also increased with age..$^{10}$ In a retrospective study of 98 elderly patients undergoing lumbar decompression and arthrodesis, Carreon et al. observed that complication rates increased with increasing age, increased blood loss, longer operative time, and number of levels fused. ${ }^{5}$ Similarly, Li et al. found that complication and mortality rates were directly related to increasing patient age. ${ }^{16}$ Accordingly, the high complication rates associated with spine surgery in this subpopula- 
TABLE 3. Total and cohort-specific differences in functional outcomes

\begin{tabular}{lccc}
\hline \multicolumn{1}{c}{ Outcome } & POSH & Non-POSH & $\begin{array}{c}\text { V } \\
\text { Value }\end{array}$ \\
\hline $\begin{array}{l}\text { Days from surgery to } \\
\text { ambulation }\end{array}$ & $1.57 \pm 0.96$ & $2.77 \pm 2.28$ & 0.02 \\
\hline $\begin{array}{l}\text { No. of feet walked on 1st } \\
\text { ambulatory day }\end{array}$ & $110.31 \pm 122.99$ & $44.92 \pm 75.38$ & 0.01 \\
\hline $\begin{array}{l}\text { No. of feet walked on day } \\
\text { of discharge }\end{array}$ & $211.61 \pm 250.23$ & $111.60 \pm 116.59$ & 0.04 \\
\hline $\begin{array}{l}\text { LOS, days } \\
\text { Discharge to home }\end{array}$ & $5.13 \pm 5.73$ & $8.72 \pm 6.10$ & 0.06 \\
\hline Discharge to SNF & 43.00 & 24.00 & 0.01 \\
\hline $\begin{array}{l}\text { Discharge to acute reha- } \\
\text { bilitation facility }\end{array}$ & 3.00 & 20.00 & 0.00 \\
\hline 30-day readmission rate & 10.00 & 8.00 & 0.77 \\
\hline 30-day mortality rate & 0.00 & 0.00 & 1.00 \\
\hline Valus are shown as the percentag & & & \\
\hline
\end{tabular}

Values are shown as the percentage or mean \pm SD unless stated otherwise.

tion of patients underscores the need for a multidisciplinary approach to manage these patients postoperatively.

A patient's discharge location after surgery is a surrogate measure of functional status. In a retrospective study of a merged database between the American College of Surgeons National Surgical Quality Improvement Program and Medicare claims between 2005 and 2008, Sacks et al. found that patients with at least 1 complication were discharged to a post-acute care facility and poor functional status was associated with increased odds of discharge to a post-acute care facility. ${ }^{19}$

Discharge to a post-acute care facility is associated with increased health care utilization. In an observational study of Medicare data on patients with a stroke or hip fracture event, Buntin et al. found that discharge to an SNF was an average of $\$ 8944$ more expensive than discharge to home. ${ }^{4}$ In a retrospective study 1127 patients enrolled in the Cardiac Arrest Registry to Enhance Survival (CARES), Chan et al. found that following cardiac arrest elderly patients who were discharged to an SNF or rehabilitation facility had a greater than 2-fold higher readmission cost compared with patients who were discharged home. ${ }^{6}$ Similarly, in a retrospective study of Medicare claims data for patients undergoing hip or knee replacements, Snow et al. found that patients reduced their health care payments by an average of $\$ 871$ by avoiding discharge to post-acute care facilities, including SNFs, home health agencies, and inpatient rehabilitation centers. ${ }^{21}$ Our study demonstrated that the POSH comanagement program led to increased discharges directly to home with a corresponding reduction in discharges to post-acute care facilities. Therefore, implementation of a postoperative comanagement program may lead to decreased health care resource utilization and costs.

To date, there has been a paucity of studies delineating the relationship between comanaged care and perioperative outcomes. In 2008, Friedman et al. described an orthopedic-geriatric comanaged program that resulted in lower-than-predicted LOS, readmission rates, complication rates, and mortality rates for patients with surgical management of hip fractures..$^{12}$ In support of these results, in a retrospective review of 758 patients undergoing surgical management with hip fractures, Kates et al. also found that LOS, 30-day readmission rates, reoperation rates, and mortality rates decreased after implementation of an orthopedic-geriatric comanaged care program..${ }^{15}$ Similarly, Kammerlander et al. described a similar orthopedic-geriatric program that resulted in decreased LOS and mortality rates, as well as an increase in return to prefracture residency, compared with before program implementation. ${ }^{14}$ Similar to these other multidisciplinary care programs, we observed that the neurosurgical-geriatric co-care POSH program was associated with a shortened in-hospital LOS and increases in the number of feet walked on ambulatory Day 1 and discharge.

This study has limitations, thereby resulting in possible implications in its interpretation. First, these data were obtained by chart review and are limited by the information recorded during care. This study is also subject to the weaknesses of retrospective analyses. The study was performed following the institution-wide implementation of the POSH program and therefore could not control for systemic changes that resulted from program implementation and may have impacted patient care. Additionally, the patient population chosen for this analysis were participants in the early implementation of the POSH program, which could have resulted in the Hawthorne effect and better outcomes. Lastly, to our knowledge this is the first program that employed a neurosurgical-geriatric postoperative comanagement program. Thus, the optimal preoperative patient selection factors that are predictive of success in such a program are unknown. Despite these limitations, the study has shown that implementing a multidisciplinary program for elderly patients undergoing lumbar spine surgery reduces perioperative complications, shortens in-hospital LOS, and improves postoperative functional status. Therefore, a comanagement program should be considered a part of the surgical management for elderly patients undergoing multilevel decompressive surgery for deformity with refractory symptoms.

\section{Conclusions}

In our experience, geriatric comanagement significantly reduces the incidence of postoperative complications, shortens in-hospital LOS, and contributes to improved perioperative functional status in elderly patients undergoing elective spinal surgery for correction of adult degenerative scoliosis.

\section{References}

1. Adogwa O, Carr RK, Kudyba K, Karikari I, Bagley CA, Gokaslan ZL, et al: Revision lumbar surgery in elderly patients with symptomatic pseudarthrosis, adjacent-segment disease, or same-level recurrent stenosis. Part 1. Two-year outcomes and clinical efficacy: clinical article. J Neurosurg Spine 18:139-146, 2013

2. Adogwa O, Owens R, Karikari I, Agarwal V, Gottfried ON, Bagley CA, et al: Revision lumbar surgery in elderly patients with symptomatic pseudarthrosis, adjacent-segment disease, 
or same-level recurrent stenosis. Part 2. A cost-effectiveness analysis: clinical article. J Neurosurg Spine 18:147-153, 2013

3. Best NM, Sasso RC: Outpatient lumbar spine decompression in 233 patients 65 years of age or older. Spine (Phila Pa 1976) 32:1135-1140, 2007

4. Buntin MB, Colla CH, Deb P, Sood N, Escarce JJ: Medicare spending and outcomes after postacute care for stroke and hip fracture. Med Care 48:776-784, 2010

5. Carreon LY, Puno RM, Dimar JR II, Glassman SD, Johnson JR: Perioperative complications of posterior lumbar decompression and arthrodesis in older adults. J Bone Joint Surg Am 85-A:2089-2092, 2003

6. Chan PS, McNally B, Nallamothu BK, Tang F, Hammill BG, Spertus JA, et al: Long-term outcomes among elderly survivors of out-of-hospital cardiac arrest. J Am Heart Assoc 5:e002924, 2016

7. Cloyd JM, Acosta FL Jr, Ames CP: Complications and outcomes of lumbar spine surgery in elderly people: a review of the literature. J Am Geriatr Soc 56:1318-1327, 2008

8. Daubs MD, Lenke LG, Cheh G, Stobbs G, Bridwell KH: Adult spinal deformity surgery: complications and outcomes in patients over age 60. Spine (Phila Pa 1976) 32:22382244, 2007

9. Devin CJ, Chotai S, Parker SL, Tetreault L, Fehlings MG, McGirt MJ: A cost-utility analysis of lumbar decompression with and without fusion for degenerative spine disease in the elderly. Neurosurgery 77 (Suppl 4):S116-S124, 2015

10. Deyo RA, Mirza SK, Martin BI, Kreuter W, Goodman DC, Jarvik JG: Trends, major medical complications, and charges associated with surgery for lumbar spinal stenosis in older adults. JAMA 303:1259-1265, 2010

11. Drazin D, Al-Khouja L, Lagman C, Ugiliweneza B, Shweikeh F, Johnson JP, et al: Scoliosis surgery in the elderly: Complications, readmissions, reoperations and mortality. J Clin Neurosci 34:158-161, 2016

12. Friedman SM, Mendelson DA, Kates SL, McCann RM: Geriatric co-management of proximal femur fractures: total quality management and protocol-driven care result in better outcomes for a frail patient population. J Am Geriatr Soc 56:1349-1356, 2008

13. Galiano K, Obwegeser AA, Gabl MV, Bauer R, Twerdy K: Long-term outcome of laminectomy for spinal stenosis in octogenarians. Spine (Phila Pa 1976) 30:332-335, 2005

14. Kammerlander C, Gosch M, Blauth M, Lechleitner M, Luger TJ, Roth T: The Tyrolean Geriatric Fracture Center: an orthogeriatric co-management model. Z Gerontol Geriatr 44:363-367, 2011

15. Kates SL, Mendelson DA, Friedman SM: Co-managed care for fragility hip fractures (Rochester model). Osteopros Int 21 (Suppl 4):S621-S625, 2010

16. Li G, Patil CG, Lad SP, Ho C, Tian W, Boakye M: Effects of age and comorbidities on complication rates and adverse outcomes after lumbar laminectomy in elderly patients. Spine (Phila Pa 1976) 33:1250-1255, 2008

17. McGirt MJ, Parker SL, Hilibrand A, Mummaneni P, Glassman SD, Devin CJ, et al: lumbar surgery in the elderly pro- vides significant health benefit in the US health care system: patient-reported outcomes in 4370 patients from the $\mathrm{N}^{2} \mathrm{QOD}$ registry. Neurosurgery 77 (Suppl 4):S125-S135, 2015

18. Ragab AA, Fye MA, Bohlman HH: Surgery of the lumbar spine for spinal stenosis in 118 patients 70 years of age or older. Spine (Phila Pa 1976) 28:348-353, 2003

19. Sacks GD, Lawson EH, Dawes AJ, Gibbons MM, Zingmond DS, Ko CY: Which patients require more care after hospital discharge? An analysis of post-acute care use among elderly patients undergoing elective surgery. J Am Coll Surg 220:1113-1121, 1121.e1-1121.e2, 2015

20. Shabat S, Arinzon Z, Gepstein R, Folman Y: Long-term follow-up of revision decompressive lumbar spinal surgery in elderly patients. J Spinal Disord Tech 24:142-145, 2011

21. Snow R, Granata J, Ruhil AV, Vogel K, McShane M, Wasielewski R: Associations between preoperative physical therapy and post-acute care utilization patterns and cost in total joint replacement. J Bone Joint Surg Am 96:e165, 2014

22. Wang MY, Green BA, Shah S, Vanni S, Levi AD: Complications associated with lumbar stenosis surgery in patients older than 75 years of age. Neurosurg Focus 14(2):e7, 2003

23. Westerveld LA, van Bemmel JC, Dhert WJ, Oner FC, Verlaan JJ: Clinical outcome after traumatic spinal fractures in patients with ankylosing spinal disorders compared with control patients. Spine J 14:729-740, 2014

24. Worley N, Marascalchi B, Jalai CM, Yang S, Diebo B, Vira S, et al: Predictors of inpatient morbidity and mortality in adult spinal deformity surgery. Eur Spine J 25:819-827, 2016

\section{Disclosures}

Dr. Karikari is a consultant for NuVasive.

\section{Author Contributions}

Conception and design: Adogwa, Elsamadicy. Acquisition of data: Elsamadicy. Analysis and interpretation of data: Adogwa, Elsamadicy. Drafting the article: Vuong. Critically revising the article: Adogwa, Elsamadicy, Vuong. Reviewed submitted version of manuscript: all authors. Approved the final version of the manuscript on behalf of all authors: Adogwa. Statistical analysis: Adogwa, Elsamadicy. Study supervision: Adogwa, Cheng, Karikari, Bagley.

\section{Supplemental Information}

\section{Previous Presentations}

Portions of this paper were presented at the joint section meeting of the American Association of Neurological Surgeons/Congress of Neurological Surgeons in Las Vegas, Nevada, March 8-11, 2017.

\section{Correspondence}

Owoicho Adogwa, Department of Neurosurgery, Rush University Medical Center, 1725 W Harrison St., Ste. 855, Chicago, IL 60612. email: owoicho.adogwa@gmail.com. 